\title{
Digital reference services
}

Ed. by Bill Katz. New York: Haworth Press, 2004. 33 I p. ISBN - 10: 0789023202

Price: US\$34.95 (soft cover)

(Published simultaneously as: The Reference Librarian, Vol. 38, Nos. 79/80, 2004.)

This book outlines the emerging field of digital referencing, introducing new issues brought on by the Internet and the role of the professional librarian. It examines the implementation of digital reference services, particularly in academic libraries, focusing on what digital referencing is, and how these services work.

Part One focuses on the basic principles, opinions and discussions of digital reference services, giving the reader a broad overview of the service, including principles and aids. Part Two examines the uses of digital referencing, based on numerous case studies in a variety of library settings, including public and academic libraries, as well as discussions on the marketing and evaluation of these digital reference services. The editor reports that digital reference services are now a standard part of reference work in most libraries. In earlier years, digital reference primarily made use of email to receive

SA Jnl Libs \& Info Sci 2006, 72(2) 
questions and provide responses. While it is still used in most services, email is often criticised for presenting barriers to two important aspects of traditional face-to- face reference services, namely, users who do not receive the same immediate response, and librarians who cannot easily conduct the reference interview that is often necessary to accurately determine and meet the user's needs. Many libraries have tried to recreate the immediacy found in face-toface reference interaction in a digital environment through the use of synchronous, real- time technologies.

However, chat technologies, which enable users to communicate on the Internet with others in real-time, have been used to provide digital reference as far back as 1995, when the Internet Public Library experimented with a text-based chat environment called a MOO (Multi-user Object Orientated). Instant messaging software products, such as AOL Instant Messenger, enable librarians to communicate in real-time with users through a series of messages sent back and forth. Instant messaging products enable librarians to indicate their availability on a contact list, share URLs and files with users, and record sessions. Instant messaging products include chat features, offering the options to communicate synchronously in a shared environment.

Why digital referencing? Users have embraced the Web and regularly turn to the Internet to find answers to their questions. Librarians, in turn have responded by placing databases, tutorials, catalogues and other tools on the Web as a way to keep current resources available to users for their research needs. Libraries have FAQs (Frequently Asked Questions), tutorials and pages where users can submit their questions via email. While these services often satisfy many user questions, none of them can provide immediate assistance at the point of need. With the availability of more tools on the Web, users are responding by accessing the library from their homes, offices, laboratories or other places besides the physical library. However, finding effective ways to reach remote users have been more difficult. Users expect answers immediately and want to get assistance at the point of need, regardless of location.

Hence, digital referencing is one of the solutions to raised user expectations. It makes assistance available to users in an easy convenient manner. Librarians are available when users have questions, and users do not have to leave their computers to get the help they need, regardless of their location or time of day. Ideally, a service of this nature should be staffed 24-hours a day, 7 days a week, but many libraries cannot afford this type of service, and thus the library must decide what hours it can be available to their patrons.

Before opening the digital reference desk to users, staff will need to be trained on the software and become familiar to answering questions in chat mode. Once the training has been completed, staff should take time to practice. Staff should be reassured that there will be unanswered questions.

Marketing is the key success of any new service. Digital referencing is not a service that is exclusively offered by libraries, hence many users turn to search engines and other Internet services before asking a reference librarian for assistance. Libraries in general should therefore identify the best methods to reach their communities. These may include talking about the new service during instructional sessions, demonstrating it to various audiences; via screensavers, and links from related web pages.

Evaluating the service is the last method guaranteeing the success of the service. Many libraries review the written transcripts to identify trends and evaluate the quality of the service. Libraries also ask users to fill out a questionnaire to get users' point of views.

This publication would be useful to all librarians and students in the field of library and information science, with its main purpose to provide access to the understanding of both theoretical and practical issues relating to digital reference services.

Reviewed by: Amina Adam, Web Librarian and Generalist, Humanities Information Services Chancellor Oppenheimer Library, University of Cape Town, Rondebosch 770I.

Tel.: 02 I 6504477

Email:amina@uctlib.uct.ac.za 